\title{
ARTICLE Group 2 innate lymphoid cells (ILC2) are regulated by stem cell factor during chronic asthmatic disease
}

\author{
Wendy Fonseca ${ }^{1}$, Andrew J Rasky ${ }^{1}$, Catherine Ptaschinski ${ }^{1}$, Susan H. Morris ${ }^{1}$, Shannon K. K. Best ${ }^{1}$, Martin Phillips ${ }^{2}$, \\ Carrie-Anne Malinczak ${ }^{1}$ and Nicholas W. Lukacs ${ }^{1}$
}

Stem cell factor (SCF) binds to the receptor c-Kit that is expressed on a number of myeloid and lymphoid cell populations, including Type 2 innate lymphoid cells (ILC2). However the importance of the SCF/c-Kit interaction in ILC2 has not been studied. Here we investigate the role of a specific SCF isoform, SCF248, in the allergic asthmatic response and SCF/c-Kit in ILC2 activation during chronic allergy. We observed that mice treated with a monoclonal antibody specific for SCF248 attenuated the development of chronic asthmatic disease by decreasing the number of mast cells, ILC2 and eosinophils, as well as reducing the accompanying pathogenic cytokine responses. These data were supported using SCF $/ / f-C o l 1-C r e-E R T$ mice and W/Wv mice that demonstrated the importance of the stem cell factor/c-Kit activation during chronic allergy and the accumulation of c-kit+ cells. Finally, these data demonstrate for the first time that SCF could activate ILC2 cells in vitro for the production of key allergic cytokines. Together these findings indicate that SCF is a critical cytokine involved in the activation of ILC2 that lead to more severe outcomes during chronic allergy and that the SCF248 isoform could be an important therapeutic target to control the disease progression.

Mucosal Immunology (2019) 12:445-456; https://doi.org/10.1038/s41385-018-0117-1

\section{INTRODUCTION}

Asthma is a chronic inflammatory disease of the airways characterized by peribronchial leukocyte accumulation accompanied by mucus overproduction and structural changes in the airway. ${ }^{1}$ These changes include airway remodeling with increased smooth muscle mass and deposition of extracellular matrix proteins that consequently narrow airways and cause bronchial hyperresponsiveness, repeated episodes of wheezing, breathlessness, and coughing. ${ }^{1,2}$ Worldwide, up to 300 million people are affected. The total cost of the disease, by medical expenses and loss of productivity, is estimated to exceed $\$ 18$ billion annually in the USA. ${ }^{1}$ In asthma, eosinophils have been reported to be a primary cell associated with the induction of bronchial mucosal injury, and further, are thought to participate in bronchial obstruction and airway hyperreactivity. ${ }^{3-5}$ Allergic inflammatory diseases are mediated by Th2 cells and Type 2 innate lymphoid cells (ILC2) that share some features, including expression of transcription factor GATA-3 which drives the production of Th2 cytokines. $^{6-8}$ ILC2 cells do not express antigen-specific receptors and are activated by epithelial-cell cytokines IL-25, IL-33 and thymic stromal lymphopoietin (TSLP). ${ }^{1,9,10}$ In these studies we show for the first time that SCF/C-Kit may be central to ILC2 activation during allergic disease and control type 2 cytokine production.

Stem cell factor (SCF) is an important cytokine involved in hematopoietic cell development and migration as well as the development of melanocytes and gametogenesis. ${ }^{11}$ SCF binds to its surface receptor, c-Kit, which is a member of the receptor tyrosine kinase family. ${ }^{11,12}$ Endogenous SCF occurs in 2 major forms, SCF220 and SCF248 (Fig. 1), with both initially found in a transmembrane form, but only the SCF248 form has an enzyme cleavable domain, which allows it to be more easily released from the surface of the cell. ${ }^{13}$ These two splice variants differ by the presence or absence of exon 6 , which encodes the enzyme cleavable domain found in the isoform SCF248. When exon 6 is removed by alternative splicing, the SCF220 isoform is inefficiently cleaved from the cell surface. ${ }^{13}$ The SCF receptor, c-Kit, is found on hematopoietic progenitor cells, melanocytes, germ cells, mast cells and eosinophils. ${ }^{11,12}$ Recent studies have identified that innate lymphoid cells, ILC2 and ILC3, express C-Kit but no study has identified the role it plays in cell activation.

Insight into the biology of SCF has been described using SI/SId mice that lack both forms of membrane-associated SCF. The SI/SId mice are runted, anemic (due to SCF's role in erythropoiesis), and have altered inflammatory/immune responses, including a reduction in allergic disease. ${ }^{14,15} \mathrm{~A}$ crucial study examined whether the two splice variants of SCF have differential biologic effects using SI/SId mutant mice transfected with either SCF248 or SCF220. The data demonstrated that SCF220 expression corrected the runting and anemia, whereas SCF248 expression did not correct the homeostatic functions but did enhance myeloid population development associated with inflammation. ${ }^{16}$ Another study that only reconstituted the SCF220 isoform (a.k.a. KL2) confirmed that animals developed normally with no runting or anemia yet had detectable defects in myeloid populations, especially mast cells. ${ }^{17}$ Together, these studies define that the splice variants have differential effects in the hematopoietic system.

In the present study, we investigated the role of the SCF248 isoform in our chronic allergic asthma model using a monoclonal antibody developed to SCF248. These data demonstrate that the allergic response was altered by targeting this single isoform and for the first time showed that SCF248 regulates ILC2 cell activation

\footnotetext{
${ }^{1}$ Department of Pathology, University of Michigan, Ann Arbor, Michigan, USA and ${ }^{2}$ Opsidio, LLC, Bryn Mawr, PA, USA
}

Correspondence: Nicholas W. Lukacs (nlukacs@umich.edu)

Received: 11 June 2018 Revised: 19 October 2018 Accepted: 16 November 2018

Published online: 7 January 2019 


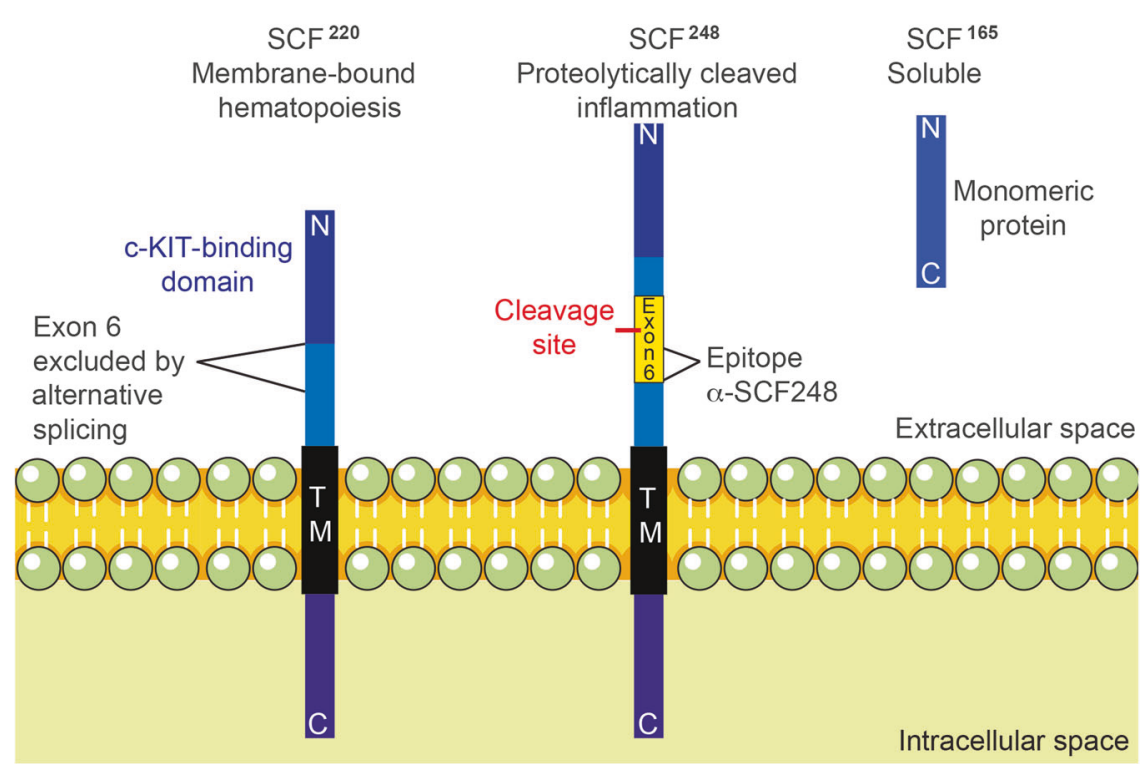

Fig. 1 SCF isoforms and $\alpha$-SCF248. Endogenous SCF is found in 2 primary forms, SCF220 and SCF248, both are initially found as transmembrane proteins, which require dimerization to activate their receptor C-KIT. SCF220 isoform is critical for hematopoiesis, while SCF248 is related to the inflammatory process. Only the SCF248 form has an enzyme cleavable domain, which allows it to be released from the surface of the cell to generate soluble SCF165 (found as a monomeric isoform that is unable to activate c-KIT in vivo ${ }^{16}$ ). These two splice variants differ by the presence or absence of exon 6 , which encodes the enzyme cleavable domain found in the isoform SCF248. Monoclonal $\alpha$-SCF248 antibodies detect an epitope on the membrane side of the cleavage in exon 6 . Thus, the monoclonal only recognizes SCF248 as an intact membrane associate protein and not the soluble SCF165 form

and that antibody targeting of SCF248 reduced the overall pathophysiology changes observed during chronic airways disease.

\section{MATERIALS AND METHODS}

Animals

All experiments involving the use of animals were approved by the University of Michigan care and use of animals committee. Female BALB/c mice, 6-8 weeks of age, were purchased from The Jackson Laboratory (Bar Harbor, ME). Mice with loxP sites on either side of exon 1 of the Kitl (kit ligand; also called SCF, stem cell factor) gene (kitL ${ }^{f / f l}$ or $S C F$ fl/fl) were purchased from Jackson Laboratory. The SCFfl/fl mice were crossed with a mouse carrying a tamoxifen-inducible Cre-recombinase [Cre-ER(T)] under the control of a regulatory sequence from the a2(I) collagen gene (C57BL/6J-Tg[Col1a2-Cre-ER(T)]) $)^{18}$ that were generously provided by Dr. Sem Phan. Both strains were on a C57BL/ 6 background. 5-7 weeks old female WBB6F1/J-Kit ${ }^{W} / K^{W}{ }^{W-v} / \mathrm{J}$ (W/ $\mathrm{W}^{\mathrm{V}}$ mice) and the congenital normal mice WBB6F1/J- Kit+/+ $[+/+]$ were purchased from The Jackson Laboratory (Bar Harbor, $\mathrm{ME}$ ). All mice were maintained under standard pathogen-free conditions.

\section{Patient samples}

All human studies were performed in accordance with an approved University of Michigan Institutional Review Board protocol after legal informed consent from adult volunteers. Subjects were recruited from the University of Michigan Asthma Clinic and had been diagnosed with asthma on the basis of clinical assessment and pulmonary testing consistent with US National Institutes of Health National Asthma Education and Prevention Program guidelines. Subjects had persistent asthma and were on daily controller medication. Healthy control subjects had not previously been diagnosed with asthma. Serum samples from patients were de-identified and SCF levels were measured using a specific ELISA (R\&D Systems, Rochester, MN).
SCF248 specific monoclonal antibody production

A peptide from exon 6 of SCF248 was generated and used as an immunogen in mice by a contract research laboratory (GenScript, Inc., Piscataway, N.J.) and hybridomas were made after several rounds of boosting immune responses. Twelve different hybridoma clones were identified as producing SCF248 peptide specific antibody and further characterized for binding and function (Fig. 1). A primary antibody with high affinity that bound to membrane SCF248 was identified, further expanded and purified to generate an endotoxin free reagent for use in our analyses, this antibody will be referred as a a-SCF248. The Mab is of the $\operatorname{lgG} 1$ isotype class. Since SCF248 exon 6 is completely conserved across mammalian species, the monoclonal antibody is fully crossreactive and binds to both mouse and human SCF248. Antibody suspended in PBS was administered to mice by intraperitoneal injection at a concentration of $20 \mathrm{mg} / \mathrm{kg}$ with a control isotype matched monoclonal antibody given at the same concentration.

Mouse chronic cockroach allergen (CRA) asthma model The allergen we used was a clinical grade, skin test CRA (Hollister Stier, Toronto, Canada) that was purified by centrifugation using Amicon Ultra-15 Centrifugal Filter Unit with Ultracel-3 membrane, 3000 MWCO (Merk Millipore, Cork, Ireland) to obtain endotoxin free CRA. Mice were sensitized intraperitoneally and subcutaneously with 500 protein nitrogen units (pnu) of CRA 1/1 in Incomplete Freund Adjuvant (Sigma-Aldrich, St. Louis, MO). Next, mice were challenged intranasally with 150 pnu of CRA on days $14,18,22$, and 26 after initial CRA sensitization to localize the response to the lung. The final two allergen challenges were given by intratracheal injection with 500 pnu on days 30 and 34. On day 35 , one day after the last allergen challenge, animals were sacrificed, and samples were taken. Balb/c mice were treated with a-SCF248 or control IgG antibodies on days 26, 29, 32, and 34 (Fig. 2a). kitL fl/fl Col1a2-Cre-ER(T $)^{+/ 0}$ mice were treated with Tamoxifen intraperitoneally (1 mg) on days 30-34 days (Fig. 2b). The same model was used in the $W / W^{V}$ mice without antibodies or tamoxifen. 
a Chronic allergic model : cockroach antigen (CRA)

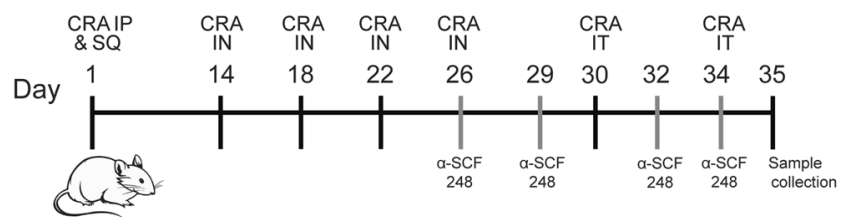

b Chronic allergic model in SCF fl/fl - Col1 Cre ${ }^{\text {ERT2 }}$ mice

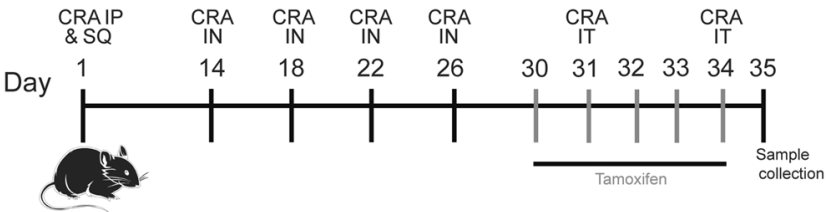

Fig. 2 Chronic allergic animal model and targeting SCF. Balb/c mice were sensitized intraperitoneally and subcutaneously with cockroach allergen (CRA) $1 / 1$ in incomplete Freund's adjuvant (IFA) and challenged intranasally with CRA on days 14, 18, 22, and 26 after initial sensitization to localize the response to the lung. The final two allergen challenges were given by intratracheal injection with four days apart on days 30 and 34 . On day 35, one day after the last allergen challenge, animals were sacrificed, and samples were taken. a Balb/c mice were treated with $\alpha$-SCF248 or lgG antibodies on 26 , 29,32 , and 34 days. b kitL ${ }^{\text {flfl }}$, Colla2-Cre-ER(T) ${ }^{+10}$ mice were treated with Tamoxifen intraperitoneally for five days 30-34 days during chronic allergic model. The same model was used in the $W / W^{v}$ mice without antibodies or tamoxifen

\section{Measurement of airway hyperreactivity}

Airway hyperreactivity was measured using mouse plethysmography, that is specially designed for the low tidal volumes (Buxco Research Systems), as previously described. ${ }^{19}$ Briefly, the mouse to be tested is anesthetized with sodium pentobarbital and intubated via cannulation of the trachea with an 18-gauge metal tube. The intubated mouse was ventilated at a volume of $200 \mu \mathrm{l}$ at a rate of 120 breaths/min. The airway resistance was measured in the closed plethysmograph by directly assessing tracheal pressure and comparing the level to corresponding box pressure changes. These values were monitored and immediately transformed into resistance measurements using computer-assisted calculations. Once baseline levels had stabilized and initial readings were taken, a methacholine challenge was given via tail vein $(0.350 \mathrm{mg} / \mathrm{kg}$ of methacholine) as previously described. ${ }^{20}$ After the methacholine challenge, the response was monitored, and the peak airway resistance was recorded as a measure of airway hyperreactivity.

Mouse lung fibroblast isolation and culture

The lungs were removed, and single cells were isolated by enzymatic digestion with $1 \mathrm{mg} / \mathrm{ml}$ collagenase A (Roche, Indianapolis, IN) and $20 \mathrm{U} / \mathrm{ml}$ DNasel (Sigma, St. Louis, MO) in RPMI 1640 containing $10 \%$ FCS. Tissues were further dispersed through an 18-gauge needle (10-ml syringe) and filtered through $100-\mu \mathrm{m}$ nylon mesh twice. Cells were centrifuged, washed, and cultured in complete medium composed of Dulbecco's modified Eagle's medium (Mediatech, Inc.) supplemented with 15\% fetal calf serum, $1 \%$ L-glutamine, and $100 \mathrm{U} / \mathrm{ml}$ penicillin, streptomycin, and fungizone (Cambrex, Walkersville, MD). Cells were used for experiments after one passage. For restimulation, lung fibroblasts were seeded after one passage into a 96-well plate. Lung fibroblasts were restimulated with a cocktail recombinant murine IL-4 (10 ng/ml; R\&D Systems, Minneapolis, USA), recombinant murine IL-13 (10 ng/ml; R\&D Systems, Minneapolis, USA), recombinant murine TNFa ( $5 \mathrm{ng} / \mathrm{ml}$; R\&D Systems, Minneapolis, USA) and recombinant human TGFb (10 ng/ml; R\&D Systems, Minneapolis, USA) for $24 \mathrm{~h}$ for gene expression analysis.
Quantitative RT-PCR

Lung tissue was homogenized in TRIzol reagent then RNA was extracted using TRIzol reagent (Invitrogen, Carlsbad, CA). CDNA was synthesized using murine leukemia virus reverse transcriptase (Applied Biosystems, Foster City, CA) and incubated at $37^{\circ} \mathrm{C}$ for 1 $h$, followed by incubation at $95^{\circ} \mathrm{C}$ for $5 \mathrm{~min}$ to stop the reaction. Real-time quantitative PCR (qPCR) was multiplexed using Taqman primers, with a FAM-conjugated probe and GAPDH with a VICconjugated probe (Applied Biosystems) to measure transcription of GATA3, T-bet, RoRc, TGFb, IL-4, IL-5, IL-9 and IL13 and cytokines receptors. Fold change was quantified using $2^{-\Delta \Delta}$ cycle threshold (CT) method. Custom primers were designed to measure Muc5ac and Gob5 mRNA levels as described. ${ }^{21}$ All reactions were run on a 7500 Real-Time PCR System (Applied Biosystems).

Lung histology

The left lung was perfused with $4 \%$ ( $\mathrm{vol} / \mathrm{vol}$ ) formaldehyde for fixation and embedded in paraffin. Five-micrometer lung sections were stained with periodic acid-Schiff (PAS) to detect mucus production, and inflammatory infiltrates. Photomicrographs were captured using a Zeiss Axio Imager Z1 and AxioVision 4.8 software (Zeiss, Munich, Germany)

\section{SCF enzyme-linked immunosorbent assays}

Murine SCF was quantified from serum samples taken from allergic mice (Fig. 1a). Serum was used to detected systemic levels of SCF using a Mouse SCF Quantikine ELISA Kit, following manufacturer's instruction (R\&D Systems, Minneapolis, MN). Human SCF was quantified from serum samples taken from asthmatic and nonasthmatic patients as a describe above, to detect systemic levels of SCF using a human SCF Quantikine ELISA Kit, following manufacturer's instruction (R\&D Systems, Minneapolis, MN).

\section{Flow cytometry}

The lungs were removed, and single cells were isolated by enzymatic digestion with $1 \mathrm{mg} / \mathrm{ml}$ collagenase A (Roche, Indianapolis, IN) and $20 \mathrm{U} / \mathrm{ml}$ DNasel (Sigma, St. Louis, MO) in RPMI 1640 containing $10 \%$ FCS. Tissues were further dispersed through an 18-gauge needle (10-ml syringe), RBCs were lysed and samples were filtered through $100-\mu \mathrm{m}$ nylon mesh twice. Cells were resuspended in PBS and live cells were identified using LIVE/DEAD Fixable Yellow Dead Cell Stain kit (Thermo Fisher Scientific, Waltham, MA), then washed and resuspended in PBS with 1\% FCS and Fc receptors were blocked with purified anti-CD16/ 32 (clone 93; BioLegend, San Diego, CA). Surface markers were identified using Abs (clones) against the following antigens, all from BioLegend: anti-Gr-1 (RB6-8C5), B220 (RA3-6B2), CD3 (145-2C11), Ter119 (Ter-119), CD11b (M1/70), CD25 (PC61), CD45 (30-F11), CD127 (SB/199), ST2 (DIH9), C-KIT (2B8) and CD90 (30-H12). SiglecF (E50-2440) was purchased from BD Biosciences (San Jose, CA). For innate lymphoid cell staining, lineage markers were anti-CD3, CD11b, B220, Gr-1, and TER119. ILC2: Lin-CD45+CD25+CD90+ ST2+c-Kit + CD127+. Eosinophils: SSC CD45+11b+SinglecF+. Mast cells: $C D 45+C D 11 b+c K I T+F c \varepsilon R 1+$. Data was collected in NovoCyte flow cytometer (ACEA Bioscience, Inc. San Diego, California). Data analysis was performed using FlowJo software (Tree Star, Oregon).

Cell sorting of ILC2 and in vitro stimulation

Lungs were removed from CRA allergic Balb/c mice (CRA allergic model as described above) and single cells were isolated by enzymatic digestion with $1 \mathrm{mg} / \mathrm{ml}$ collagenase A (Roche, Indianapolis, IN) and $20 \mathrm{U} / \mathrm{ml}$ DNasel (Sigma, St. Louis, MO) in RPMI 1640 containing $10 \%$ FCS. Tissues were further dispersed through an 18-gauge needle (10-ml syringe) and filtered twice through a 100$\mu \mathrm{m}$-nylon mesh filter. Red blood cells were lysed by the addition of $12 \mathrm{ml}$ of ice cold sterile water followed by the addition of $12 \mathrm{ml}$ ice cold $1.8 \% \mathrm{NACl}$ to restore osmolality. A portion of the single cell suspension was used to isolated CD4+ cells using microbeads 

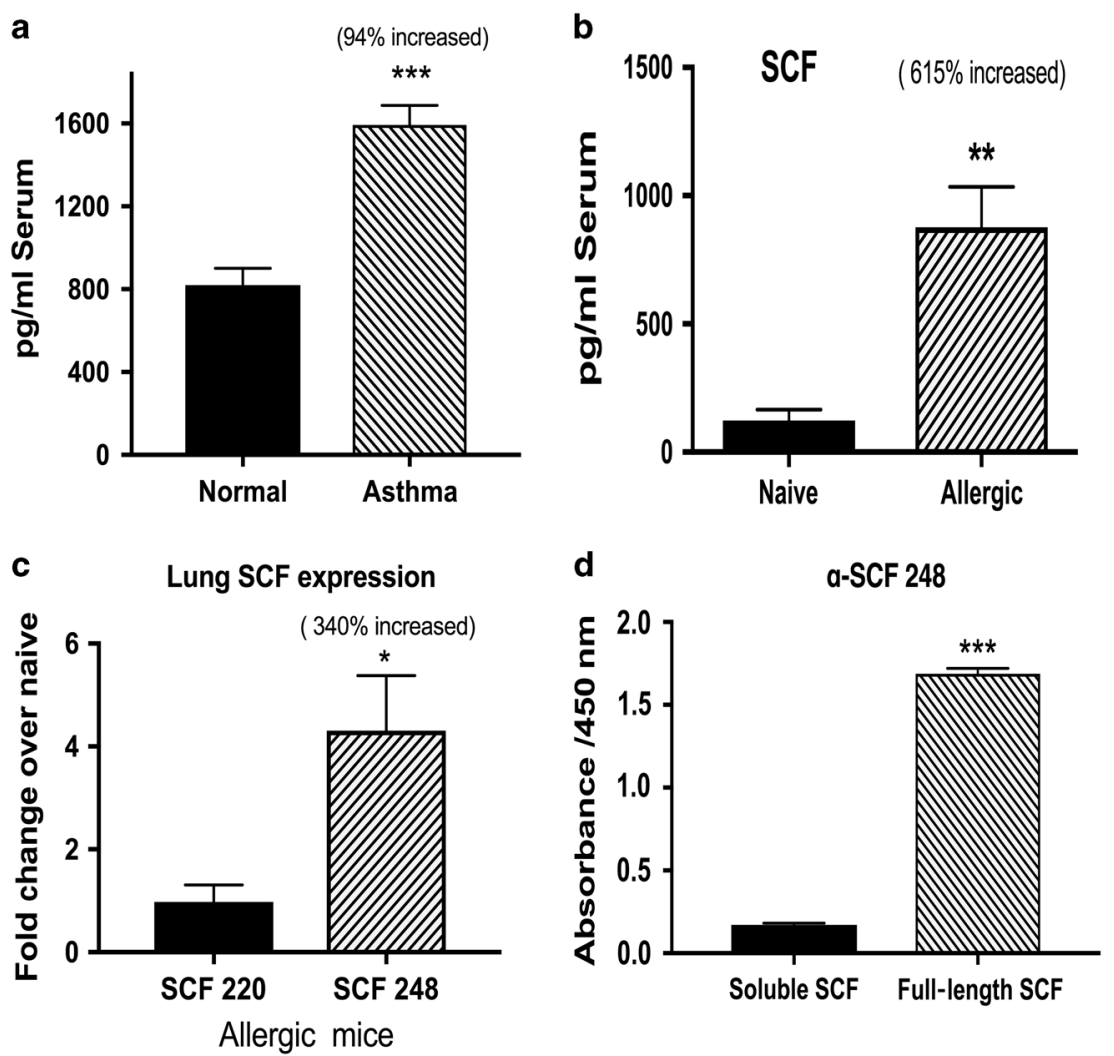

Fig. 3 SCF expression during allergy and differential expression of 248SCF isoform. a Level of soluble (ECD) SCF in serum samples of asthmatic patients $(n=9)$ and non-asthmatics $(n=8)$ was measured by ELISA. b Soluble SCF in the serum of chronic allergic mice or naïve mice was measured by ELISA. c qPCR expression of the two isoforms SCF220 and SCF 248 in the lungs of allergic and naïve mice. d A monoclonal antibody against 248SCF was made using a synthetic peptide from exon 6 . specifically binds to the full-length recombinant SCF protein that contains exon 6 but not the cleaved extracellular domain of SCF as indicated by direct ELISA. Data represents mean \pm SE from each experiment with $4-5$ replicates per experiment in the mouse studies. All data are representative of two independent experiments. ${ }^{*} p<0.05$, ${ }^{* *} p<0.01,{ }^{* * *} p<0.001$

(Miltenyi Biotec, Bergisch Gladbach, Germany). Single cells isolated were used for magnetic depletion of mature hematopoietic cells, including T cells, B cells, monocytes/macrophages, granulocytes and erythrocytes and their committed precursors using The Lineage Cell Depletion Kit (Miltenyi Biotec, Bergisch Gladbach, Germany) following manufacturer's instruction. The isolated linage negative cells were used to select CD45 positive cells using CD45 microbeads (Miltenyi Biotec, Bergisch Gladbach, Germany) following manufacturer's instruction. The enriched cells that were Lin-, CD45+ were used for single-cell sorting in the Sony Biotechnology iCyt Synergy SY3200 BSC Cell Sorter at the Flow cytometry core at the University of Michigan. CD90+ST2+cells were sorted with $>93 \%$ efficiency. ILC2 cells were cultured in complete RPMI 1640 with or without recombinant murine IL-2 $(10 \mathrm{ng} / \mathrm{ml} ; \mathrm{R} \& D$ Systems, Minneapolis, USA). ILC2 cells were cultured in a 96 round well plate that was previously coated over night with murine SCF $(1 \mu \mathrm{g} /$ $\mathrm{ml}$ in PBS overnight, then washed twice before adding cells), and stimulated with recombinant murine SCF $(50 \mathrm{ng} / \mathrm{ml}$; R\&D Systems, Minneapolis, USA) in culture, in the presence or absence recombinant murine IL-25 $(20 \mathrm{ng} / \mathrm{ml}$; R\&D Systems, Minneapolis, USA) for $24 \mathrm{~h}$ for gene expression analysis.

\section{Statistical analysis}

Data were analyzed by Prism 6 (GraphPad Software). Data presented are mean values \pm SEM. Comparison of two groups was performed with an unpaired, two-tailed Student $t$-test. Comparison of three or more groups was analyzed by ANOVA with a Tukey posttest. A $p$-value $<0.05$ was considered significant.

\section{RESULTS}

SCF expression during allergy and differential expression of 248SCF isoform

To investigate the participation of SCF in chronic asthma, we measured levels of soluble SCF in serum samples and found significantly increased SCF in the serum of asthmatic patients compared with the non-asthmatic controls (Fig. 3a). We also analyzed soluble SCF in the serum of chronic allergic or naïve mice, and we observed a similar effect with increased levels of SCF in the serum of allergic mice compared with naïve mice (Fig. 3b). To further investigate the role of the different SCF isoforms, we analyzed the expression of the two isoforms, SCF220 and SCF248, in the lungs of allergic mice. The SCF248 isoform was overexpressed in the lungs of allergic mice, whereas the expression level of the SCF220 isoform was unchanged compared to levels in naïve mice (Fig. $3 c$ ). Thus, the increased circulating levels of SCF in the serum of allergic animals was likely due to the increased lung expression of SCF248.

In order to further study the SCF248 isoform, we generated a specific Mab against a synthetic peptide from exon 6 that is found only in SCF248. Our target peptide was derived from exon 6 expressed on the membrane cell surface side of the enzyme cleavage site. Thus, the a-SCF248 Mab would only bind to membrane-associated SCF248 protein and not to soluble SCF extracellular domain (ECD) or SCF220 (Fig. 1). The specific binding was confirmed by direct ELISAs that showed the a-SCF248 Mab detected the full-length recombinant SCF248 protein but not the soluble ECD of SCF protein (Fig. 3d). The exon 6 sequence is 

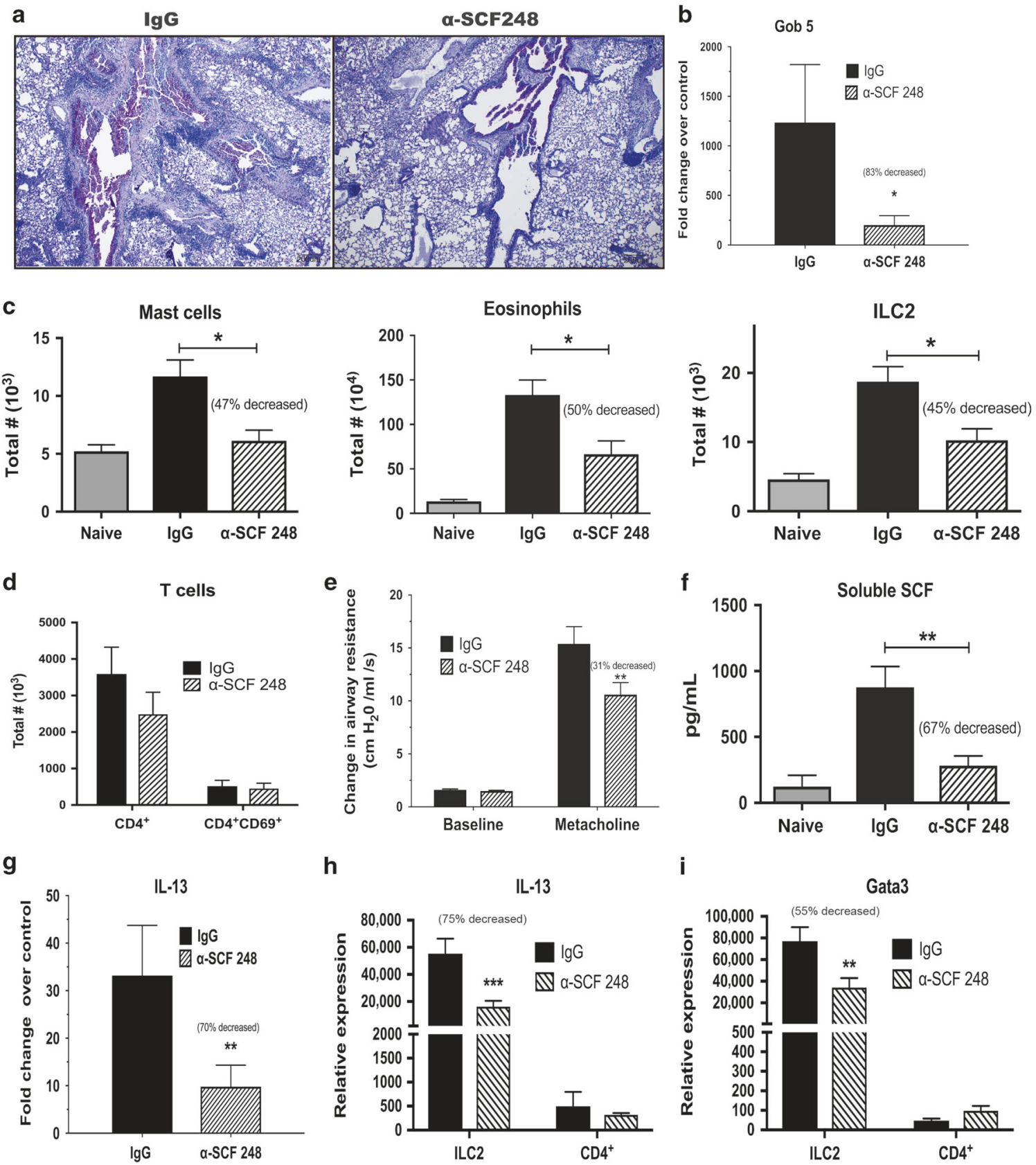

Fig. $4 \alpha$-SCF248 attenuates chronic pulmonary disease in mouse model. Chronic allergen challenged animals were treated by systemic administration of the $\alpha$-SCF248 during the last four allergen challenges and attenuated disease severity was observed as indicated by a Lung histologic examinations PAS stain for mucus detection, shows decreased mucus deposition in the mice treated with $\alpha$-SCF248 compare with IgG. b qPCR of mucus-related gene Gob5. c Flow cytometry assessed lung mast cells, Eosinophils and ILC2. d Flow cytometry T cells. e Airway hyperactivity AHR. f Serum extracellular domain of SCF measured by ELISA was reduced in the anti-SCF248 treated animals. $\mathbf{g}$ qPCR of lung cytokine IL-13 expression demonstrated decreased allergic inflammation. $\mathbf{h}$ IL-13 expression in Sorted ILC2 and CD4+ cells from allergic mice, and $\mathbf{i}$ Gata 3 expression relative to GAPDH control gene expression. Together these data reflect the importance of the isoform SCF248 for the progression of chronic allergic disease. Data represent mean $\pm \mathrm{SE}$, from 4 to 5 mice/group. All data are representative of two to three independent experiments. ${ }^{*} p<0.05,{ }^{* *} p<0.01,{ }^{* *} p<0.001$

conserved in mouse and human and is not found in SCF220 isoform.

a-SCF248 Mab attenuates chronic pulmonary disease

To study the participation of the SCF248 isoform in the allergic airway response, we probed our chronic allergy mouse model with a-SCF248 Mab. Chronic allergic animals were treated systemically with a-SCF248 Mab during the last week of airway allergen challenge (Fig. 2a). The lung histology of treated mice demonstrated that the a-SCF248 Mab reduced the levels of inflammatory cell accumulation and the mucus deposition in the airways (Fig. 4a). We corroborated these data by quantifying mucus related genes by qPCR (Fig. 4b) where we observed significantly decreased expression of gob5 gene in the lungs of mice treated with a-SCF248 Mab compared to the control treated mice. To better characterize the pathology and to add objective quantitative data to our histological observations, whole lungs were dispersed, and the leukocyte numbers were examined. The 
a-SCF248 Mab treated mice had significant decreases in the numbers of mast cells, eosinophils and ILC2 cells (c-Kit+ cells) (Fig. 4C). In contrast, CD4+ T cells, a source of Th2 cytokines, were not reduced by anti-SCF248 treatment (Fig. 4d). Airway hyperreactivity (AHR) is a characteristic feature of asthmatic lung disease and was significantly decreased in the group of mice that were treated with a-SCF248 Mab (Fig. 4e). The treatment with a-SCF248 Mab also decreased the level of soluble SCF in the blood of the mice (Fig. 4f). The expression of IL-13 in whole lungs of a-SCF248 Mab treated mice was significantly decreased compared with the control allergic mice (Fig. $4 \mathrm{~g}$ ). To further examine the relative contribution of ILC2 compared to Th cells we isolated the individual cells from lungs of allergic mice after the final challenge and assessed Th2 cytokines. The data indicate that while ILC2 have decreased IL-13 and decreased Th2 associated transcription factor GATA3 after anti-SCF treatment, no significant changes were observed by Th cells (Fig. 4h, i). Interestingly, anti-SCF did not significantly alter IL-4, IL-5, or IL-9 in either the ILC2 or Th cell populations (Supplementary Fig. 1). These findings are significant as high levels of soluble SCF are correlated with asthma severity in humans ${ }^{22}$ and in our studies SCF was also increased in patients with persistent asthma (Fig. 3a). Together, these data demonstrate diminished lung pathology in the mice that were treated with aSCF248 Mab and included decreased mast cells, eosinophils and ILC2 in the lung, as well as decreased airway mucus and Th2 cytokine expression.

Pulmonary fibroblasts grown from chronic allergen challenged animals are altered by the treatment of a-SCF248 Mab

As previously described, fibroblasts grown from chronically inflamed lung tissue have an altered phenotype compared to those grown from non-inflamed tissue. ${ }^{23}$ Importantly, previous studies have shown that fibroblasts make predominantly SCF248 and not the SCF220 isoform. ${ }^{24}$ To examine whether neutralization of SCF248 has an impact on the expression of fibrotic genes in pulmonary fibroblasts, levels of fibronectin and collagen 3a1 (COL3A1) mRNA were analyzed. The a-SCF248 Mab treatment mediated a reduction in the expression of these genes in whole lung mRNA compared to control treated allergic animals (Fig. 5a). To specifically examine the fibroblast phenotype, lungs from animals chronically challenged with allergen were dispersed, and fibroblasts were grown from them as previously described. ${ }^{25}$ Separate fibroblasts lines were developed from each mouse and the analysis was performed after a single passage in vitro when $>95 \%$ of the cells were fibroblasts (not shown). qPCR analysis demonstrated that fibroblasts from lungs of chronic allergen treated mice had a significant increase in COL3A1 at baseline compared to fibroblasts from naïve lungs (Fig. 5b) that was further increased upon pro-fibrotic cytokine activation, including IL-4, IL-13, TGF $\beta$ and TNF compared to untreated fibroblasts (Fig. 5c). In fibroblasts grown from lungs of allergic animals treated with a-SCF248 Mab, the expression of the COL3A1 gene was not significantly increased compared to normal lung fibroblasts even under pro-fibrotic cytokine activation (Fig. 5b, c). These observations suggest that controlling the degree of airway inflammation had an impact on the fibroblast phenotype and that the severity of the pulmonary disease was controlled by blockade of the SCF248 membrane associated isoform. When fibroblasts from allergic mice were isolated and the expression of SCF220 and SCF248 compared to expression in fibroblasts from non-allergic mice and only the SCF248 (not SCF220) was significantly increased (Fig. 5d). When pulmonary fibroblasts were activated by IL-4, IL-13, and TGF $\beta$ they preferentially up regulated the SCF248 isoform and not SCF220 (Fig. 5e) with IL13 promoting the highest expression of SCF248. These latter data further reflect earlier published data of predominate expression of SCF248 by fibroblasts. ${ }^{17}$
Alteration of chronic allergic responses in $\mathrm{SCF}^{\mathrm{fl} / \mathrm{fl}}-\mathrm{Col} 1 \mathrm{CreRT}$ mice that lack SCF specifically in fibroblasts

It has been described that SCF is highly expressed in fibroblasts grown from lungs of chronic allergic mice. ${ }^{25}$ In addition, the above data indicate that fibroblasts preferentially express SCF248 with little increase in SCF220. We corroborated the importance of SCF during chronic allergy and the significance of fibroblast SCF expression using the $\mathrm{SCF}^{\mathrm{fl} / \mathrm{fl}}$-Col1 $\mathrm{Cre}+\mathrm{RT}$ mice that specifically lack SCF in fibroblasts (Fig. 6a). Since these mice have a deletion of SCF in only Col1 + fibroblasts after treatment with tamoxifen, they allow us to understand the impact of SCF in fibroblasts during the allergic responses. We induced deletion of SCF by intraperitoneal injections of tamoxifen during the last week of allergen challenge (Fig. 2b), a timeframe similar to the a-SCF248 Mab treatment. We observed that $\mathrm{Cre}+\mathrm{SCF}^{\mathrm{f} / \mathrm{fl}}$ animals had a statistically significant with a $31 \%$ decrease in AHR in response to methacholine challenge relative to change of control mice (Fig. 6b) accompanied by reduced histopathology, with low mucus deposition in the airways compared with the Cre- SCF ${ }^{f / f l}$ mice (Fig. $6 \mathrm{c}$ ). When lung leukocytes were analyzed by flow cytometry, a reduction in cKit + eosinophils and ILC2 cells was observed (Fig. $6 \mathrm{~d}$ ). These data indicate that fibroblast specific deletion of SCF during the chronic phase of allergic disease leads to an improved pathology suggesting that the fibroblasts are a primary source of SCF248 in the lung during chronic allergic/asthmatic responses.

SCF receptor mutant $\mathrm{W} / \mathrm{W}^{\mathrm{v}}$ mice define the role of the eosinophil and ILC2 cells

Mutant mice (SI/SId) that produce lower levels of soluble SCF compared to WT mice and have no membrane-associated SCF, also have an altered allergen-induced response, including decreased AHR and peribronchial eosinophilia (11). To further investigate the role of the SCF/c-Kit activation and extend our studies during chronic allergy, we used W/Wv mice, that have truncated and point-mutated c-Kit W (white spotting locus or cKit) and $W_{v}$ alleles, respectively. These mutations generate reduced c-Kit tyrosine kinase-dependent signaling that results in mast cells deficiency, impaired melanogenesis and result in anemia and sterility. ${ }^{26}$ Thus, these mice can be used to test the importance of the SCF/c-Kit activation during allergic response. We observed that the W/Wv mice had significantly lower responses to the allergen challenges including decreased airway leukocyte infiltration and mucus deposition (Fig. 7a), significantly reduced expression of muc5ac and gob5 mucus related genes (Fig. 7b), and reduced expression of Th2 cytokines IL-4, IL-5, and IL13 in the lung (Fig. 7c). Further, ILC2 cells were virtually absent from these mice and there was a significant decrease in mast cells and eosinophils in the lung compared to allergic congenital normal mice (Kit+/+) (Fig. 7d). These data demonstrate a critical role of SCF/c-Kit activation during chronic allergic disease for the accumulation of mast cells, eosinophils and ILC2 into the lung that contribute to enhanced pathology during allergic disease.

SCF mediated ILC2 cell activation in vitro

We observed in the previous experiments that a-SCF248 Mab decreased the number of c-Kit + cells in the lung during allergic disease, and although it is known that ILC2 are c-Kit+, there are no studies regarding whether ILC2 can be activated via the c-Kit ligand, SCF, to produce Th2 cytokine. ILC2 were sorted from a lineage negative CD45+ enriched cell population from the lung of allergic mice by flow cytometry (Fig. 8a). Sorted lung ILC2 were analyzed by qPCR for the expression of transcription factors T-bet, Gata-3, and Rorc which are differentially expressed in ILC1, ILC2, and ILC3 cells. ${ }^{27}$ The high levels of GATA3 in our culture confirmed that the sorted cells were ILC2 (Fig. 8b). To evaluate the effect of SCF directly to the ILC2, we stimulated the sorted cells in vitro for $16 \mathrm{~h}$ with or without recombinant murine SCF (rSCF), and qPCR was performed to detect GATA3 and ID2, two transcription factors 
a

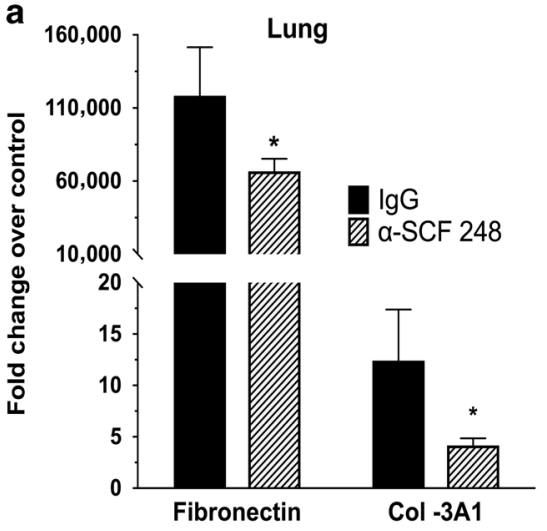

c

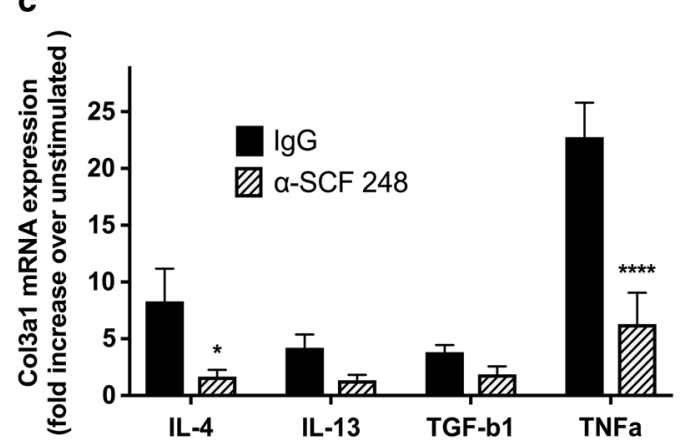

b
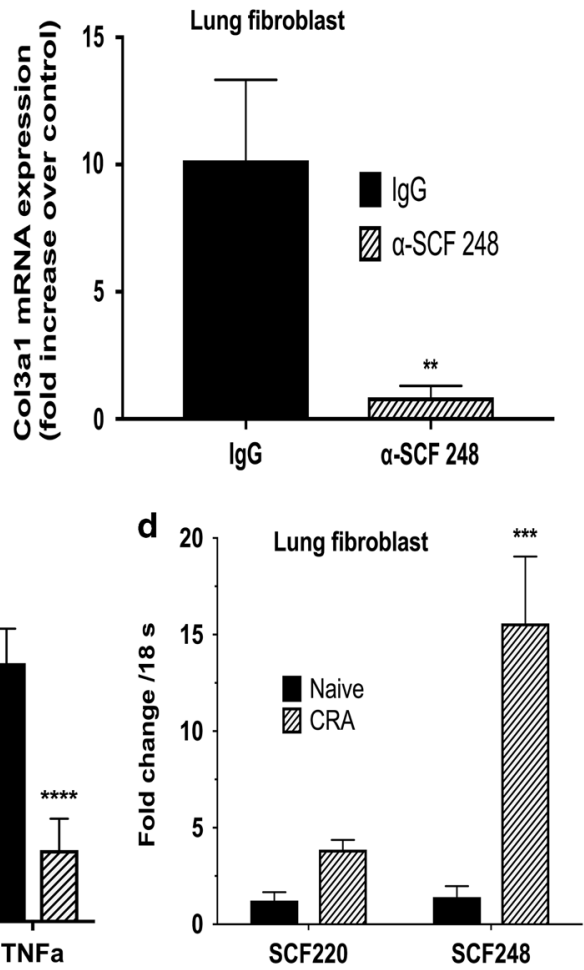

e

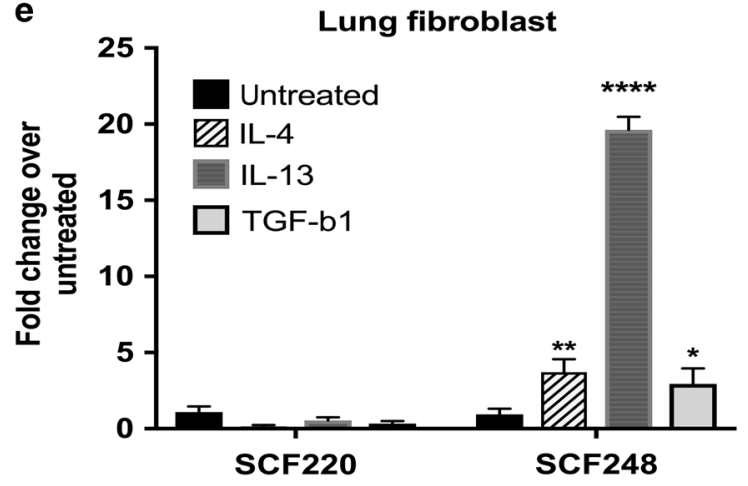

Fig. 5 Pulmonary fibroblast grown from chronic allergen mice are altered by the treatment of $\alpha$-SCF248. Fibrotic phenotype in the lung of the $\alpha$-SCF248 treated mice was reduced as observed by (a) qPCR in lung tissue to measure expression of remodeling genes fibronectin and collagen 3a1. b Fibroblasts grown from lungs of allergic antibody treated mice cultured at baseline (c) or stimulated by profibrotic cytokines IL-4, IL-13, TNFa, and TGFb1. The cytokine stimulated fibroblasts are calculated by the fold increased expression of Col3a1 over the baseline measurement. $\mathbf{d}$ The expression of the differential mRNA expression of SCF220 and SCF248 isoforms in the lungs of naïve and chronic allergen (CRA) challenge animals. e Activation of lung fibroblasts by profibrotic cytokines promote SCF248 expression but not SCF220. Data represent mean \pm SE from 5 mice/group. All data are representative of two to three independent experiments. ${ }^{*} p<0.05,{ }^{* *} p<0.01,{ }^{* * *} p<0.001$

essentials for the differentiation and maintenance of ILC2. We observed that the cells stimulated with SCF significantly increased the expression of GATA3 and inhibitor of DNA binding 2 (ID2) (Fig. 8c, d), suggesting the SCF is participating in the development of ILC2 and maintenance of phenotype.

To further examine the role of SCF activation for promoting ILC2 effector functions, the sorted cells were cultivated in vitro for $24 \mathrm{~h}$ in media (+IL-2) with or without rSCF and the cytokine expression was analyzed by qPCR. We observed that ILC2 cells incubated with rSCF expressed significantly increased levels of IL-4, IL-5, II-9, IL-13 and TGFb compared with ILC2 cells in media alone (Fig. 8e). Expression of IL-17 or IFNg was not detected (data not shown). We also observed significantly increased expression of IL-9r and IL-25r (IL-17RB) receptors in the cells treated with rSCF compared with the control treated cells (Fig. 8f). Since we previously showed that our model of chronic allergic disease was associated with IL-25 production, ${ }^{28}$ and because we observed increased expression of IL-25r, we stimulated the ILC2 cells with murine recombinant IL-25 (rIL-25) that activates ILC2 cells to produce Th2 cytokines. ${ }^{29}$ As expected, the rIL-25 stimulated ILC2 cells showed significantly increased levels of IL-5, IL-9, and IL-13. Further, when both SCF and IL-25 were added together there was an increase in the same cytokines compared with the single cytokine treatments (Fig. 8g). This increase appeared to be additive for IL-5 and synergistic for IL-9 and IL-13. These data indicated SCF could be a critical cytokine for the development and maintenance of ILC2 and further amplify IL-25 for the activation of ILC2 for cytokine expression in the lung during chronic allergic responses. 
a

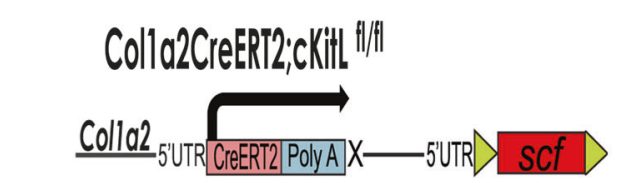

b Cre -

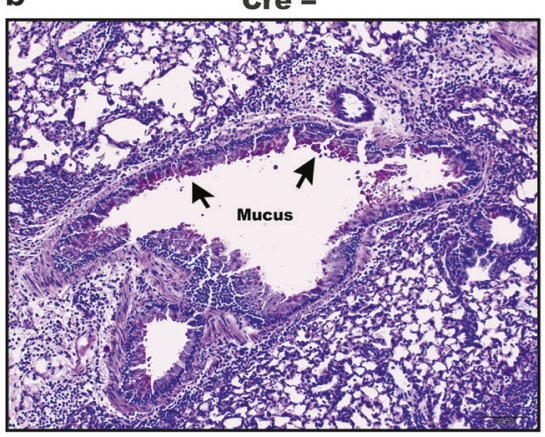

Cre +

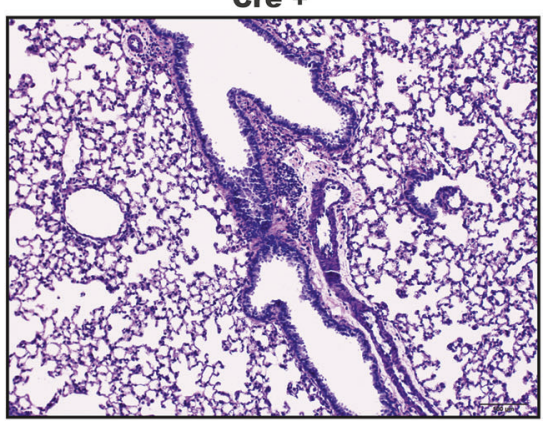

ILC2

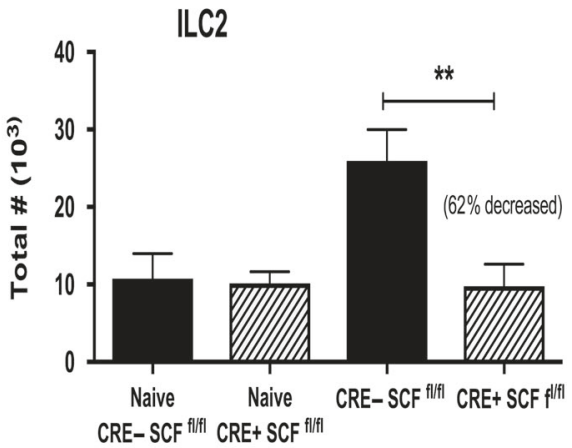

Fig. 6 The chronic allergen-induced pulmonary disease is attenuated in SCFfl/fl-Col1-Cre mice (a) Cre gene under the promotor of Col1 generated the SCFfl/fl-Col1-Cre mice that do not express SCF in fibroblasts when Cre is induced by tamoxifen intraperitoneally. The deletion of fibroblast SCF attenuates the allergen-induced pathology, including (b) airway inflammation by histopathology PAS staining for mucus detection, $\mathbf{c} A H R, \mathbf{d})$ and reduces the numbers of c-Kit + eosinophils and ILC2 cells in the lungs. Data represent mean \pm SE from each group with 5 mice/group. All data are representative of three independent experiments. ${ }^{*} p<0.05,{ }^{* *} p<0.01,{ }^{* * *} p<0.001$

\section{DISCUSSION}

In this study, we demonstrated that the SCF248 isoform has a predominant effect on the overall allergen-induced environment during airway inflammation and for the first time show that SCF is critical for ILC2 cell expansion and/or function. While our studies do not completely rule out direct effects of SCF248 depletion on $T$ cells, there were not decreases in pulmonary $T$ cell numbers or apparent differences in total Th2 cytokine mRNA from isolated pulmonary $T$ cells. The functional difference between the two major isoforms of SCF, 220AA, and 248AA was identified in studies that transfected the isoforms in Sld mutant animals that lack both forms of transmembrane SCF. ${ }^{16}$ The studies demonstrated that the 220AA form was responsible for homeostatic functions and the 248AA form for induction of mast cells. Furthermore, the transfection of SCF248 into keratinocytes in Sld mice led to mastocytosis and significant inflammation in skin, whereas SCF220 transfection corrected normal melanocyte growth and function without the development of mastocytosis. ${ }^{14}$ These latter studies extend earlier findings that showed that SCF220, and not SCF248, is required for normal erythropoiesis and overall health of the SId mice ${ }^{30}$ further indicating that SCF220 predominantly functions as a homeostatic factor. Our data have further confirmed the link between SCF248 and inflammatory disease as experiments blocking SCF248 during allergen-induced responses led to a significant reduction in the severity of airway disease. Previous data from our lab and others have identified that SCF and its receptor c-Kit appear to have important activation functions during chronic allergic disease, ${ }^{31-37}$ but these studies are the first to show that a preferential isoform has a significant impact. SCF may also represent an important biomarker of disease. The clinical data show that SCF was highly expressed in lungs of severe asthmatics ${ }^{38}$ and the increased circulating levels of SCF correlate with the severity of disease. ${ }^{22,31}$ In this work we showed that allergic mice have increased SCF in serum compared to control naïve mice and that a-SCF248 Mab treatment decreases serum SCF levels, correlating with the reduced airway disease and release into the circulation. Altogether, these studies suggest that an isoform-specific antibody against SCF248 can provide a protective effect in the development of chronic asthmatic disease. 
a WBB6F1-Kit $+/+[+/+]$ CRA

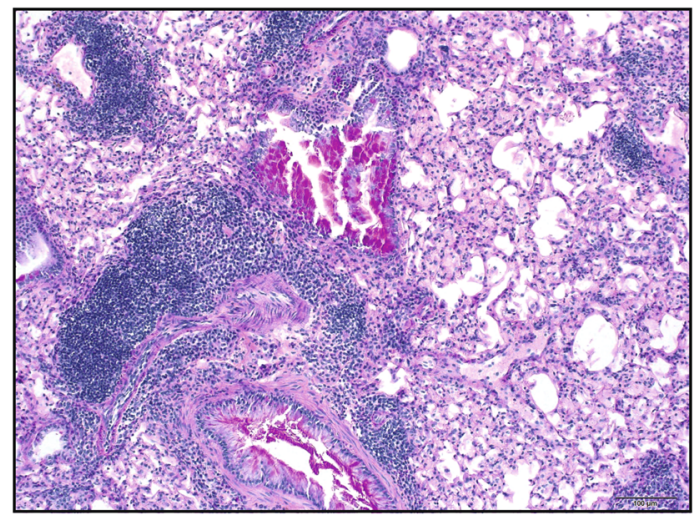

b

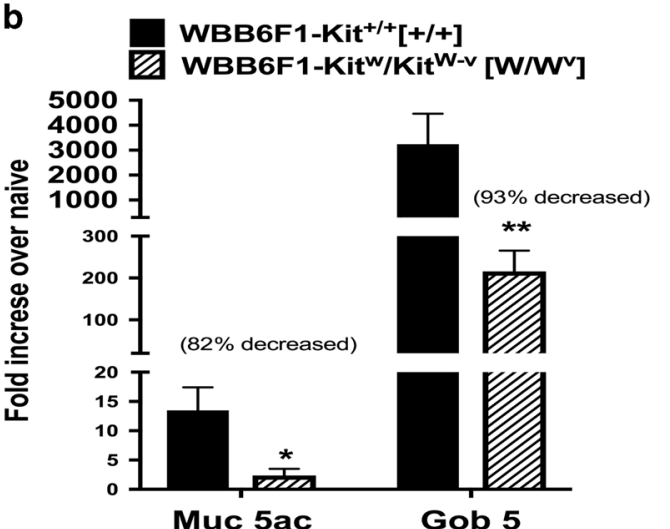

WBB6F1-Kitw/KitW-v [W/Wv] CRA

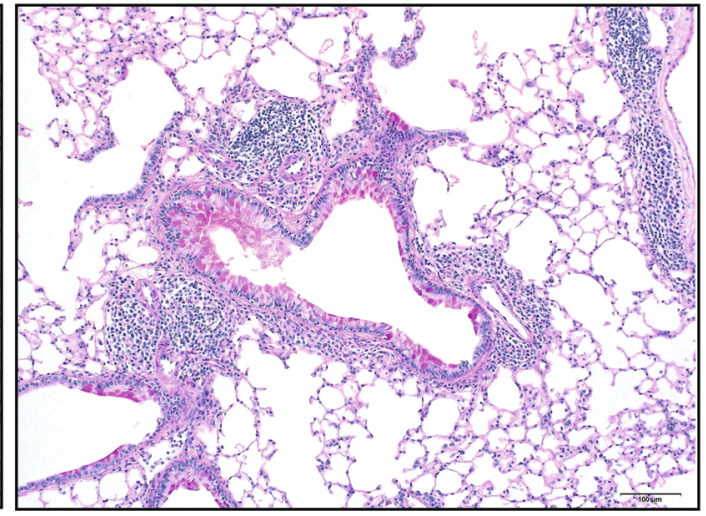

C

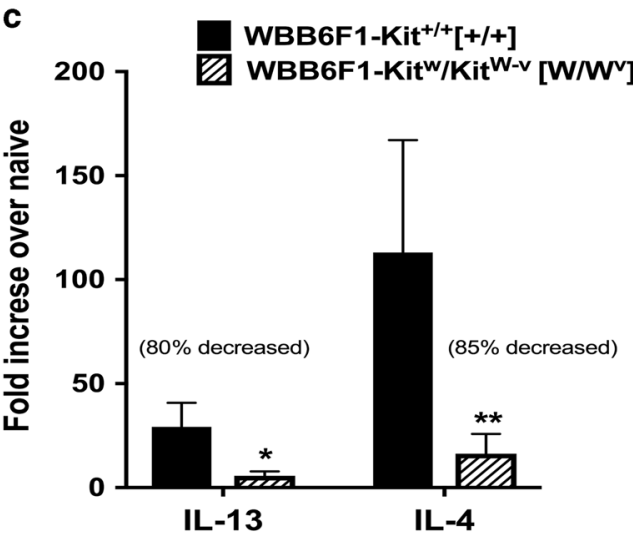

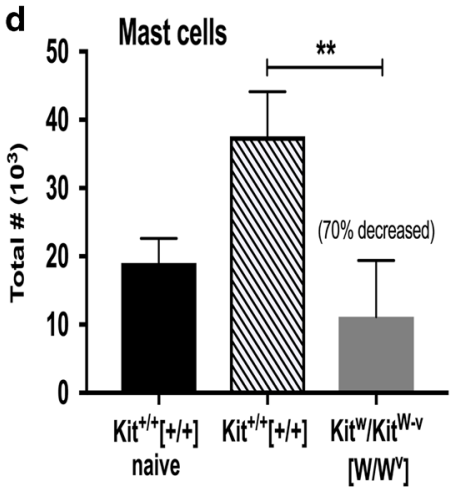
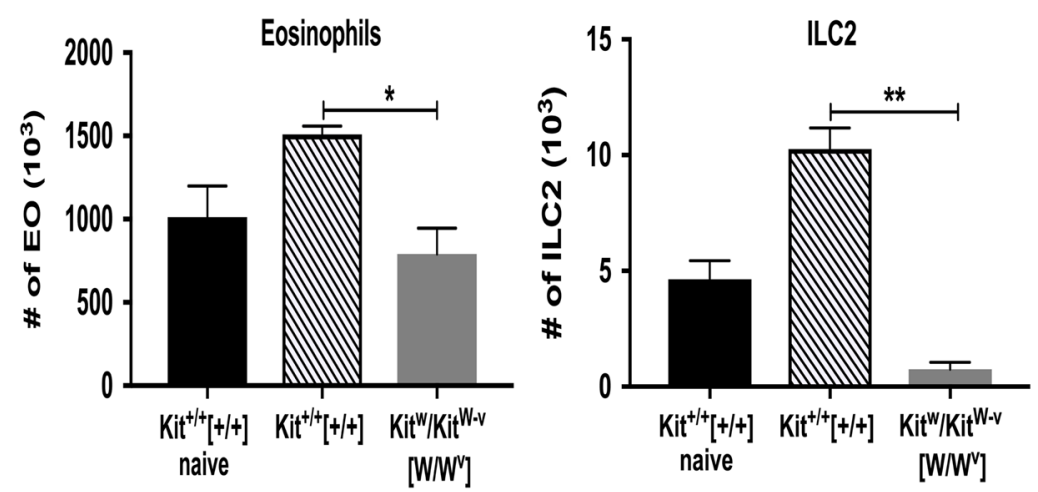

Fig. 7 SCF receptor mutant W/W $\mathrm{W}^{\vee}$ mice demonstrated the important role of the SCF-c-Kit activation during chronic allergic diseases. The lack of c-Kit signaling reduces the allergen-induced (a) airway inflammation and mucus deposition in airway by histopathology, PAS staining (b) qPCR of mucus-related genes Muc5 and Gob5, c qPCR of lung cytokines IL-4 and IL-13 expression, $\mathbf{d}$ decreased numbers of Mast cells, Eosinophils and ILC2 in the lung. Data represent mean \pm SE from each group with 4 to 5 mice/group. All data are representative of two independent experiments. ${ }^{*} p<0.05,{ }^{* *} p<0.01,{ }^{* *} p<0.001$

Using the specific antibodies to SCF248 and genetically altered animals we were able to identify critical c-Kit+ immune populations associated with severe allergic disease. In particular, SCF-c-Kit activation was important in the accumulation of eosinophils, mast cells, and ILC2 cells in the lung during allergic disease. SCF directly activates eosinophils ${ }^{39,40}$ causing these cells to degranulate and induce pro-fibrotic mediators, leukotrienes and chemokines, as well as promote the survival of eosinophils independent of IL-5. ${ }^{40}$ SCF is long been established as a mast cell differentiation, survival and activation factor, capable of contributing the mast cell induced responses in disease. ${ }^{41,42}$ Current studies show that SCF is also an activator of ILC2 cells to produce Th2 cytokines and creating an environment that promotes allergic disease. A causative role of c-Kit+ cells during lung inflammation and remodeling has been suggested using a murine model of Aspergillus infection and allergy, ${ }^{43}$ by IL-9-driven mast cells expansion of CD25 + ILC2 cells and led to exacerbated allergic inflammation by c-Kit dependent ILC2 cell cytokines. In the present study, W/Wv mice (c-kit mutant) had decreased numbers of mast cells, eosinophils and a near absence of ILC2 cells in the lung supporting the importance of the SCF-c-Kit interaction in these cell populations during allergic disease. SCF appeared to especially drive IL-9 levels in ILC2 cells. IL-9 can recruit mast cells to the airways, induce goblet cell hyperplasia, ${ }^{44}$ provides a positive feedback loop that amplified ILC2 cytokine production, ${ }^{43,45}$ and increases proliferation of ILC2. ${ }^{6-8}$ Importantly, ILC2 can be directly 
$\mathbf{a}$

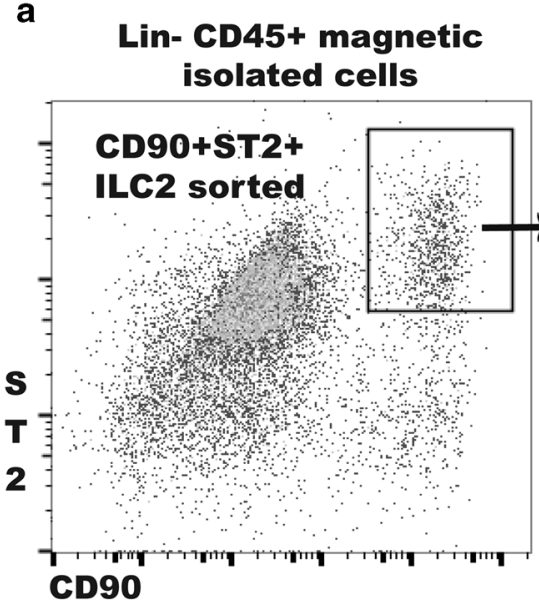

b

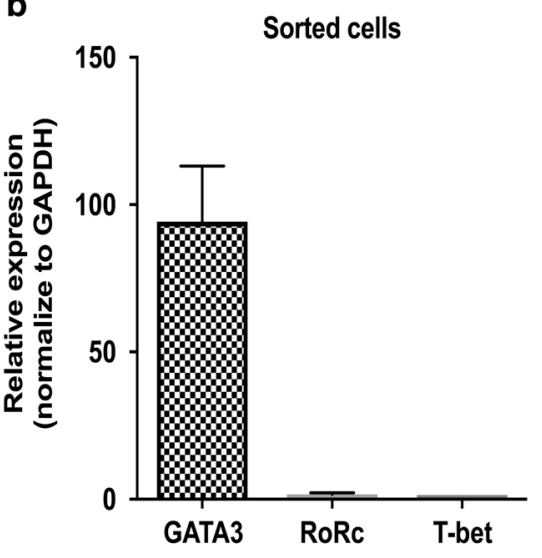

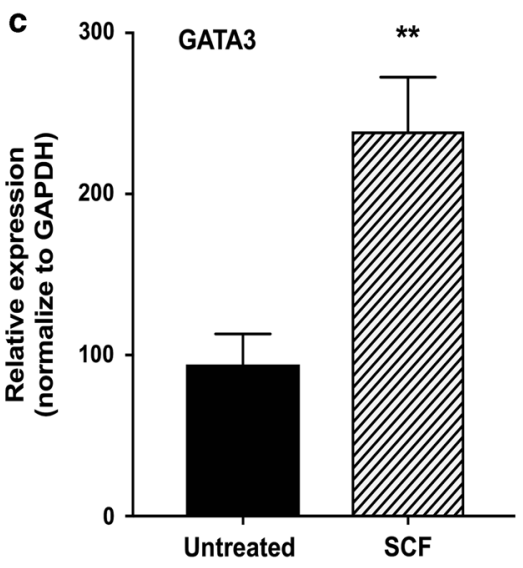

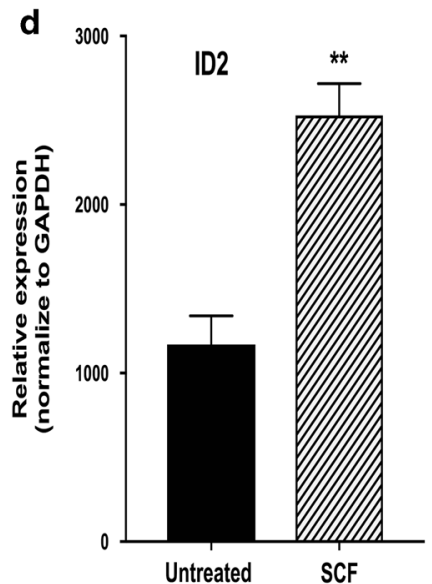

g

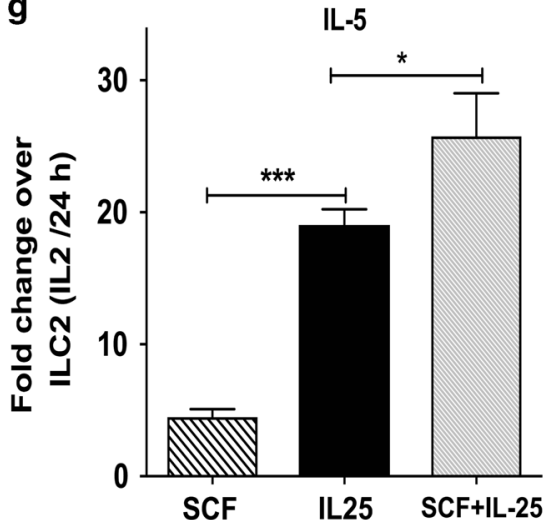

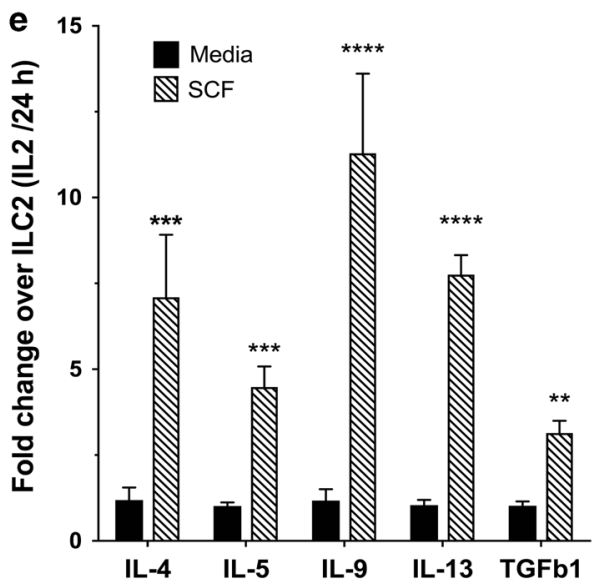

IL-9

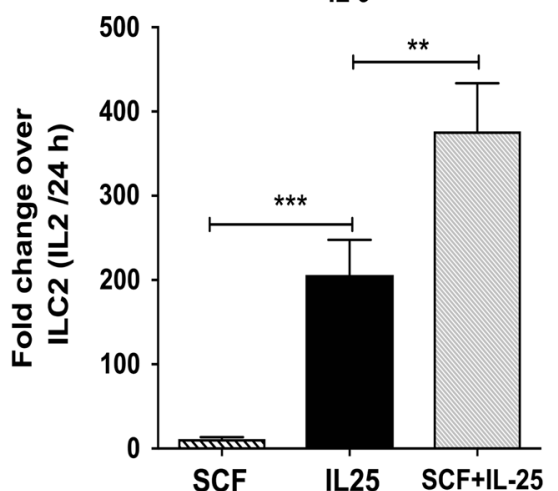

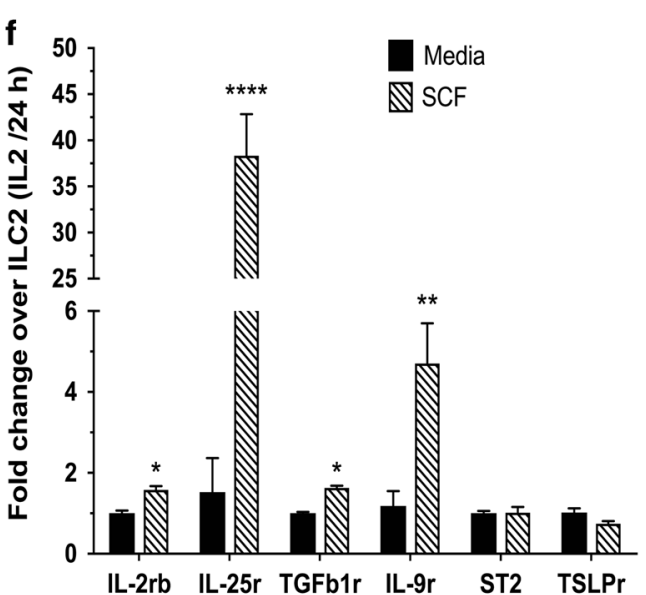

IL-13

Fig. 8 SCF mediated ILC2 activation in vitro. a ILC2 were sorted from a Lin- CD45+ enriched cells population from the lung of allergic mice by flow cytometry, $\mathbf{b}$ Identity of the sorted cells was analyzed by qPCR to detected transcription factors GATA3, RoRc, and T-bet. c Sorted cells were cultured in vitro for $16 \mathrm{~h}$ in untreated media (RPMI) with or without murine SCF (rSCF) and qPCR was performed to detect GATA3 (d) and ID2 (e) Sorted cells were cultured in vitro for $24 \mathrm{~h}$ alone or in presences of recombinant murine SCF ( $\mathrm{rSCF}$ ), and the cytokine expression was analyzed by qPCR, c Expression of cytokine receptors by qPCR in SCF stimulated ILC2, f ILC2 cultured with SCF and/or IL-25 (rlL-25) were analyzed by qPCR for expression of cytokines. Data represent mean \pm SE of 4 replicates/experiment. All data are representative of three independent experiments. ${ }^{*} p<0.05,{ }^{* *} p<0.01,{ }^{* * *} p<0.001,{ }^{* * * *} p<0.0001$

activated by SCF to produced Th2 cytokines (IL-4, IL-5, IL-9, and IL13). Interestingly, we detected increased expression of IL-4. IL-4 + ILC2 have been reported by others, ${ }^{46,47}$ and the expression of IL-4 by ILC2 has been linked with the expression of IL-25. ${ }^{9}$ Here, we observed that SCF can work together with other cytokines including IL-25 to co-activate ILC2 cells for additive or synergistic cytokine production. The fact that SCF also upregulated IL-25R (IL$17 \mathrm{RB})$ and IL-9R suggests that SCF/c-kit may promote an enhanced Th2 response that activates the ILC2 in the presence of IL-25 and/or IL-9. Thus, a major aspect of blocking SCF248 in our studies has linked the ability to alter the function and activation of ILC2 cells to the pathogenic allergic responses. ${ }^{24}$

It is reported that SCF is required for the differentiation of ILC since SCF is necessary in cell cultures for the in vitro differentiation of ILC from common lymphoid progenitors. ${ }^{48,49}$ Nevertheless, the mechanisms have not been clearly described whether SCF is 


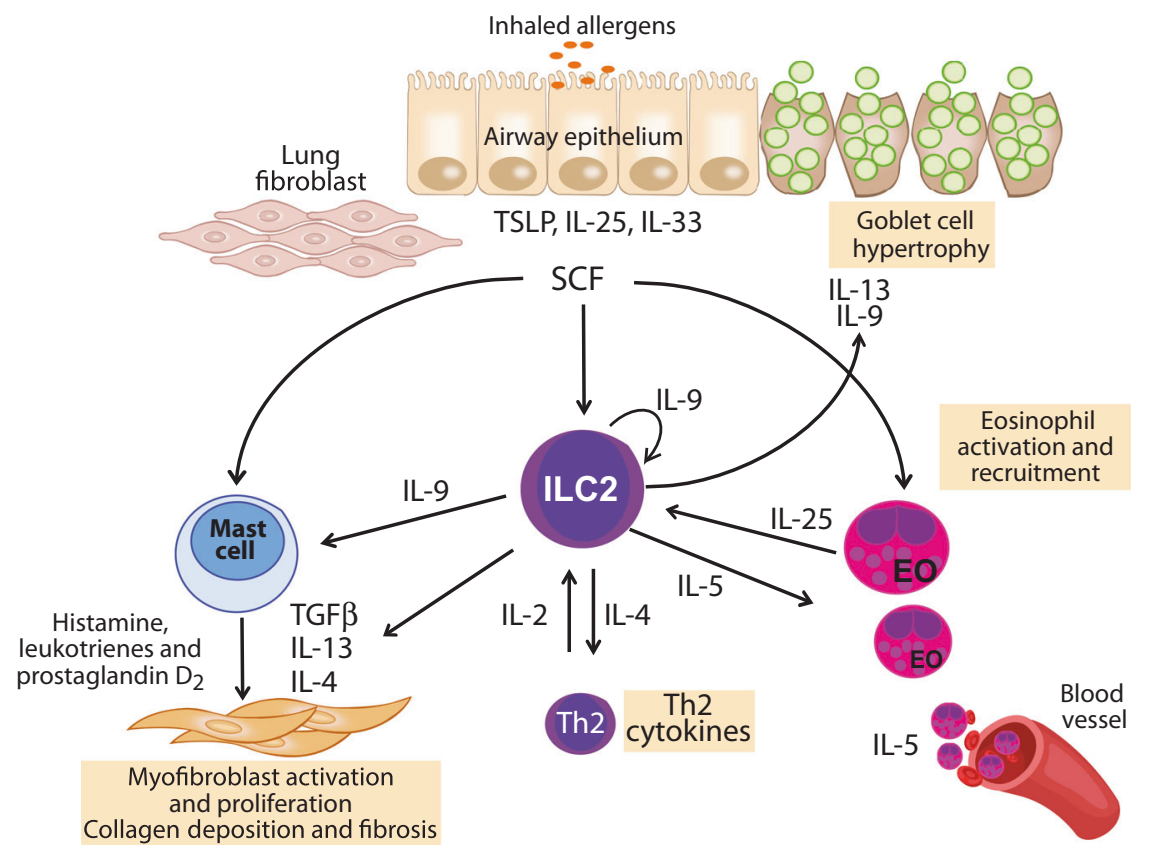

Fig. 9 Hypothetical model for the function of SCF in the regulation of ILC2 cells. SCF is expressed by lung fibroblast during chronic allergy, together with lung epithelial cytokines such as IL-25 activate lung ILC2. Activation of ILC2 along with activation of mast cells and eosinophils by SCF leads to enhanced IL- 9 and IL-13 production that generates goblet cell hypertrophy and mucus production. IL- 9 enhances the survival and/or proliferation of ILC2 as well as the production of IL-5 and IL-13 that together leads to an allergic immune environment

involved during the differentiation or activation of ILC2. In this work, we demonostrated that the stimulation of isolated ILC2 with SCF increased the expression of GATA3, a transcription factor that has been described that controls cell fate and maintenance of type 2 innate lymphoid cells. ${ }^{27}$ We also observed that SCF increased the expression of ID2 in the sorted ILC2. ID2 is a critical transcription factor required for ILC development, ${ }^{50,51}$ and a high expression of ID2 has been observed in progenitor cells that have committed their fates to an ILC lineage. ${ }^{52}$ Future detailed studies will be necessary to determine whether SCF is fundamental for the development of the ILC from CLP, and specifically for the differentiation of ILC2.

SCF is expressed by fibroblasts almost exclusively in the SCF248 isoform ${ }^{17}$ as confirmed by our data. c-Kit + cells are associated with induction and amplification of bronchial mucosal injury, and are thought to participate in bronchial obstruction and airway hyperreactivity. $3,6,43$ Since ILC2 cells have been linked with Th2 responses in the lung, ${ }^{6-8}$ and express c-Kit receptor ${ }^{6-8}$ the relationship between the myofibroblast activation and ILC2 cells is logical. Using the SCF ${ }^{\mathrm{f} / \mathrm{fl}}$-Col1 CreRT mice SCF that were specifically deleted in collagen 1 producing cells (i.e., fibroblasts) during the chronic stage of the allergic model the data showed a decreased allergic response that was similar to the specific anti-SCF248 Mab treatment. Importantly, this included a reduction in pulmonary Th2 cytokine levels and a significant reduction in c-Kit + ILC2 cells and eosinophils. Thus, we provide novel data that suggest that ILC2 cells promote a pathologic response during chronic allergic disease through the activation by SCF248 isoform that is the predominant isoform produced by fibroblasts, as well as other cell populations in the lung during allergic asthmatic disease.

In conclusion, SCF is a critical cytokine that plays a pivotal role in the allergic environment as depicted in Fig. 9 that integrates other critical allergic cytokines, including IL-9 and IL-25, and is involved in the activation of eosinophils, mast cells and now ILC2 that lead to more severe chronic lung allergy/asthma. These responses likely enhance the Th2 environment leading to both direct and indirect effects on T cell driven allergic disease. Furthermore, the identification of the SCF248 varient provides the first data that a specific isoform corresponds to the severity of allergic responses (Fig. 9). Altogether, these studies highlight that SCF248 may be a selective therapeutic target that is involved in the severity of allergic airways disease at least partially through a novel mechanism of activation of ILC2.

\section{ACKNOWLEDGEMENTS}

This work was funded in part by NIH grants Al036302 and HL138013. We thank Judith Connett, PhD for editorial review and Gabriela Monzon for artwork.

\section{ADDITIONAL INFORMATION}

The online version of this article (https://doi.org/10.1038/s41385-018-0117-1) contains supplementary material, which is available to authorized users.

Competing interests: The authors declare no competing interests.

\section{REFERENCES}

1. Lambrecht, B. N. \& Hammad, H. The immunology of asthma. Nat. Immunol. 16, 45-56 (2015).

2. Maddox, L. \& Schwartz, D. A. The pathophysiology of asthma. Annu. Rev. Med. 53, 477-498 (2002).

3. Oliveira, S. H. et al. Stem cell factor induces eosinophil activation and degranulation: mediator release and gene array analysis. Blood 100, 4291-4297 (2002).

4. O'Byrne, P. M., Naji, N. \& Gauvreau, G. M. Severe asthma: future treatments. Clin. Exp. Allergy. 42, 706-711 (2012)

5. McBrien, C. N. \& Menzies-Gow, A. The biology of Eosinophils and their role in asthma. Front. Med. 4, 93 (2017).

6. McKenzie, A. N. Type-2 innate lymphoid cells in asthma and allergy. Ann. Am. Thorac. Soc. 11(Suppl 5), S263-S270 (2014).

7. Barlow, J. L. et al. Innate IL-13-producing nuocytes arise during allergic lung inflammation and contribute to airways hyperreactivity. J. Allergy Clin. Immunol. 129, 191-198 (2012). e191-194.

8. Halim, T. Y., Krauss, R. H., Sun, A. C. \& Takei, F. Lung natural helper cells are a critical source of Th2 cell-type cytokines in protease allergen-induced airway inflammation. Immunity 36, 451-463 (2012).

9. Fallon, P. G. et al. Identification of an interleukin (IL)-25-dependent cell population that provides IL-4, IL-5, and IL-13 at the onset of helminth expulsion. J. Exp. Med. 203, 1105-1116 (2006). 
10. Camelo, A. et al. IL-33, IL-25, and TSLP induce a distinct phenotypic and activation profile in human type 2 innate lymphoid cells. Blood Adv. 1, 577-589 (2017).

11. Galli, S. J., Tsai, M. \& Wershil, B. K. The c-kit receptor, stem cell factor, and mast cells. What each is teaching us about the others. Am. J. Pathol. 142, 965-974 (1993).

12. Ashman, L. K. The biology of stem cell factor and its receptor C-kit. Int. J. Biochem. Cell. Biol. 31, 1037-1051 (1999).

13. Broudy, V. C. Stem cell factor and hematopoiesis. Blood 90, 1345-1364 (1997).

14. Williams, D. A. \& Majumdar, M. K. Analysis of steel factor (stem cell factor) isoforms in the hematopoietic microenvironment. Stem Cells 12(Suppl 1), 67-74 (1994). discussion75-67.

15. Broxmeyer, H. E. et al. The kit receptor and its ligand, steel factor, as regulators of hemopoiesis. Cancer Cells 3, 480-487 (1991).

16. Kapur, R. et al. Signaling through the interaction of membrane-restricted stem cell factor and c-kit receptor tyrosine kinase: genetic evidence for a differential role in erythropoiesis. Blood 91, 879-889 (1998).

17. Tajima, Y. et al. Consequences of exclusive expression in vivo of Kit-ligand lacking the major proteolytic cleavage site. Proc. Natl Acad. Sci. USA 95, 11903-11908 (1998).

18. Zheng, B., Zhang, Z., Black, C. M., de Crombrugghe, B. \& Denton, C. P. Liganddependent genetic recombination in fibroblasts: a potentially powerful technique for investigating gene function in fibrosis. Am. J. Pathol. 160, 1609-1617 (2002).

19. Campbell, E., Hogaboam, C., Lincoln, P. \& Lukacs, N. W. Stem cell factor-induced airway hyperreactivity in allergic and normal mice. Am. J. Pathol. 154, 1259-1265 (1999).

20. Dolgachev, V., Petersen, B. C., Budelsky, A. L., Berlin, A. A. \& Lukacs, N. W. Pulmonary IL-17E (IL-25) production and IL-17RB + myeloid cell-derived Th2 cytokine production are dependent upon stem cell factor-induced responses during chronic allergic pulmonary disease. J. Immunol. 183, 5705-5715 (2009).

21. Miller, A. L., Bowlin, T. L. \& Lukacs, N. W. Respiratory syncytial virus-induced chemokine production: linking viral replication to chemokine production in vitro and in vivo. J. Infect. Dis. 189, 1419-1430 (2004).

22. Makowska, J. S., Cieslak, M. \& Kowalski, M. L. Stem cell factor and its soluble receptor (c-kit) in serum of asthmatic patients- correlation with disease severity. BMC Pulm. Med. 9, 27 (2009).

23. $L i$, Y. et al. Severe lung fibrosis requires an invasive fibroblast phenotype regulated by hyaluronan and CD44. J. Exp. Med. 208, 1459-1471 (2011).

24. Huang, E. J., Nocka, K. H., Buck, J. \& Besmer, P. Differential expression and processing of two cell associated forms of the kit-ligand: KL-1 and KL-2. Mol. Biol. Cell 3, 349-362 (1992).

25. Dolgachev, V., Berlin, A. A. \& Lukacs, N. W. Eosinophil activation of fibroblasts from chronic allergen-induced disease utilizes stem cell factor for phenotypic changes. Am. J. Pathol. 172, 68-76 (2008).

26. Grimbaldeston, M. A. et al. Mast cell-deficient W-sash c-kit mutant Kit W-sh/W-sh mice as a model for investigating mast cell biology in vivo. Am. J. Pathol. 167, 835-848 (2005).

27. Hoyler, T. et al. The transcription factor GATA-3 controls cell fate and maintenance of type 2 innate lymphoid cells. Immunity 37, 634-648 (2012).

28. Petersen, B. C., Budelsky, A. L., Baptist, A. P., Schaller, M. A. \& Lukacs, N. W. Interleukin-25 induces type 2 cytokine production in a steroid-resistant interleukin-17RB + myeloid population that exacerbates asthmatic pathology. Nat. Med. 18, 751-758 (2012).

29. Saenz, S. A. et al. IL25 elicits a multipotent progenitor cell population that promotes $\mathrm{T}(\mathrm{H}) 2$ cytokine responses. Nature 464, 1362-1366 (2010).

30. Bodine, D. M., Orlic, D., Birkett, N. C., Seidel, N. E. \& Zsebo, K. M. Stem cell factor increases colony-forming unit-spleen number in vitro in synergy with interleukin6, and in vivo in SI/Sld mice as a single factor. Blood 79, 913-919 (1992).
31. Lei, Z. et al. SCF and IL-31 rather than IL-17 and BAFF are potential indicators in patients with allergic asthma. Allergy 63, 327-32 (2008).

32. Da Silva, C. A. et al. Marked stem cell factor expression in the airways of lung transplant recipients. Respir. Res. 7, 90 (2006).

33. Berlin, A. A., Hogaboam, C. M. \& Lukacs, N. W. Inhibition of SCF attenuates peribronchial remodeling in chronic cockroach allergen-induced asthma. Lab. Invest. 86, 557-565 (2006).

34. Da Silva, C. A. \& Frossard, N. Regulation of stem cell factor expression in inflammation and asthma. Mem. Inst. Oswaldo. Cruz. 100(Suppl 1), 145-151 (2005).

35. Berlin, A. A., Lincoln, P., Tomkinson, A. \& Lukacs, N. W. Inhibition of stem cell factor reduces pulmonary cytokine levels during allergic airway responses. Clin. Exp. Immunol. 136, 15-20 (2004).

36. Oliveira, S. H. \& Lukacs, N. W. Stem cell factor: a hemopoietic cytokine with important targets in asthma. Curr. Drug. Targets Inflamm. Allergy 2, 313-318 (2003).

37. Columbo, M. et al. The human recombinant c-kit receptor ligand, rhSCF, induces mediator release from human cutaneous mast cells and enhances IgE-dependent mediator release from both skin mast cells and peripheral blood basophils. J. Immunol. 149, 599-608 (1992).

38. Al-Muhsen, S. Z., Shablovsky, G., Olivenstein, R., Mazer, B. \& Hamid, Q. The expression of stem cell factor and c-kit receptor in human asthmatic airways. Clin. Exp. Allergy. 34, 911-916 (2004).

39. Yuan, O. Austen, K. F., Friend, D. S., Heidtman, M. \& Boyce, J. A. Human peripheral blood eosinophils express a functional c-kit receptor for stem cell factor that stimulates very late antigen 4 (VLA-4)-mediated cell adhesion to fibronectin and vascular cell adhesion molecule 1 (VCAM-1). J. Exp. Med. 186, 313-323 (1997).

40. Dolgachev, V., Thomas, M., Berlin, A. \& Lukacs, N. W. Stem cell factor-mediated activation pathways promote murine eosinophil CCL6 production and survival. J. Leukoc. Biol. 81, 1111-1119 (2007).

41. Huang, B. et al. SCF-mediated mast cell infiltration and activation exacerbate the inflammation and immunosuppression in tumor microenvironment. Blood 112, 1269-1279 (2008).

42. Ito, T. et al. Stem cell factor programs the mast cell activation phenotype. J. Immunol. 188, 5428-5437 (2012).

43. Moretti, S. et al. A mast cell-ILC2-Th9 pathway promotes lung inflammation in cystic fibrosis. Nat. Commun. 8, 14017 (2017).

44. Vermeer, P. D., Harson, R., Einwalter, L. A., Moninger, T. \& Zabner, J. Interleukin-9 induces goblet cell hyperplasia during repair of human airway epithelia. Am. J. Respir. Cell Mol. Biol. 28, 286-295 (2003).

45. Wilhelm, C. et al. An IL-9 fate reporter demonstrates the induction of an innate IL9 response in lung inflammation. Nat. Immunol. 12, 1071-1077 (2011).

46. Doherty, T. A. et al. Lung type 2 innate lymphoid cells express cysteinyl leukotriene receptor 1 , which regulates $\mathrm{TH} 2$ cytokine production. J. Allergy Clin. Immunol. 132, 205-213 (2013).

47. Pelly, V. S. et al. IL-4-producing ILC2s are required for the differentiation of $\mathrm{TH} 2$ cells following Heligmosomoides polygyrus infection. Mucosal Immunol. 9, 1407-1417 (2016).

48. Baerenwaldt, A. et al. Flt3 ligand regulates the development of innate lymphoid cells in fetal and adult mice. J. Immunol. 196, 2561-2571 (2016).

49. Seehus C. \& Kaye J. In vitro differentiation of murine innate lymphoid cells from common lymphoid progenitor cells. Bio. Protoc. 6, (2016).

50. Verykokakis, M., Zook, E. C. \& Kee, B. L. ID'ing innate and innate-like lymphoid cells. Immunol. Rev. 261, 177-197 (2014).

51. Zhong, C. \& Zhu, J. Transcriptional regulators dictate innate lymphoid cell fates. Protein Cell 8, 242-254 (2017).

52. Klose, C. S. N. et al. Differentiation of type $1 \mathrm{ILCs}$ from a common progenitor to all helper-like innate lymphoid cell lineages. Cell 157, 340-356 (2014). 